\title{
Modulated Amplitude Waves and the Transition from Phase to Defect Chaos
}

\author{
Lutz Brusch, ${ }^{1}$ Martín G. Zimmermann, ${ }^{2}, *$ Martin van Hecke, ${ }^{1,3}$ Markus Bär, ${ }^{1}$ and Alessandro Torcini ${ }^{4}$ \\ ${ }^{1}$ Max-Planck-Institut für Physik komplexer Systeme, Nöthnitzer Straße 38, D-01187 Dresden, Germany \\ ${ }^{2}$ Instituto Mediterraneo de Estudios Avanzados, IMEDEA (CSIC-UIB), E-07071 Palma de Mallorca, Spain \\ ${ }^{3}$ Center for Chaos and Turbulence Studies, The Niels Bohr Institute, Blegdamsvej 17, 2100 Copenhagen, Denmark \\ ${ }^{4}$ Istituto Nazionale di Fisica della Materia, Unità di Firenze, Largo Enrico Fermi 2, I-50125 Firenze, Italy
}

(Received 31 January 2000)

\begin{abstract}
The mechanism for transitions from phase to defect chaos in the one-dimensional complex GinzburgLandau equation (CGLE) is presented. We describe periodic coherent structures of the CGLE, called modulated amplitude waves (MAWs). MAWs of various periods $P$ occur in phase chaotic states. A bifurcation study of the MAWs reveals that for sufficiently large period, pairs of MAWs cease to exist via a saddle-node bifurcation. For periods beyond this bifurcation, incoherent near-MAW structures evolve towards defects. This leads to our main result: the transition from phase to defect chaos takes place when the periods of MAWs in phase chaos are driven beyond their saddle-node bifurcation.
\end{abstract}

PACS numbers: 47.54.+r, 05.45.-a

Spatially extended systems can exhibit, when driven away from equilibrium, irregular behavior in space and time: this phenomenon is commonly referred to as spatiotemporal chaos [1]. The one-dimensional complex Ginzburg-Landau equation (CGLE),

$$
\partial_{t} A=A+\left(1+i c_{1}\right) \partial_{x}^{2} A-\left(1-i c_{3}\right)|A|^{2} A,
$$

describes pattern formation near a Hopf bifurcation and has become a popular model to study spatiotemporal chaos [1-13]. As a function of $c_{1}$ and $c_{3}$, the CGLE exhibits two qualitatively different spatiotemporal chaotic states known as phase chaos (when $A$ is bounded away from zero) and defect chaos (when the phase of $A$ displays singularities where $A=0$ ). The transition from phase to defect chaos can either be hysteretic or continuous; in the former case, it is referred to as $L_{3}$, in the latter as $L_{1}$ (Fig. 1). Despite intensive studies [5-13], the phenomenology of the CGLE and, in particular, its "phase" diagram [5,7] are far from being understood. Moreover, it is under dispute whether the $L_{1}$ transition is sharp, and whether a pure phase chaotic (i.e., defect-free) state can exist in the thermodynamic limit [9].

It is the purpose of this paper to elucidate these issues by presenting the mechanism which creates defects in transient phase chaotic states. Our analysis consists of four parts: (i) We describe a family of modulated amplitude waves (MAWs), i.e., pulselike coherent structures with a characteristic spatial period $P$. (ii) A bifurcation analysis of these MAWs reveals that their range of existence is limited by a saddle-node (SN) bifurcation. For all $c_{1}, c_{3}$ within a certain range, we define $P_{\mathrm{SN}}$ as the period of the MAW for which this bifurcation occurs. (iii) We show that for $P>P_{\mathrm{SN}}$, i.e., beyond the $\mathrm{SN}$ bifurcation, near-MAW structures display a nonlinear evolution to defects. It is found that, in phase chaos, near MAWs with various $P$ 's are created and annihilated perpetually.

The transition to defect chaos takes place when nearMAWs with $P>P_{\mathrm{SN}}$ occur in a phase chaotic state. (iv) Finally, instabilities to splitting of, respectively, interaction between MAWs are identified as the relevant processes which locally decrease, respectively, increase $P$ in phase chaos. We will argue that the $\mathrm{SN}$ curve for $P \rightarrow \infty$ is a lower bound (see Fig. 1) for the transition from phase chaos to defect chaos.

From a general viewpoint, our analysis shows that there is no collective behavior that drives the transition. Instead, strictly local fluctuations drive local structures beyond their $\mathrm{SN}$ bifurcation and create defects.

(i) MAWs as coherent structures. - By coherent structures we mean uniformly propagating structures of the form [11-13]

$$
A(x, t)=a(x-v t) e^{i \phi(x-v t)} e^{-i \omega t},
$$

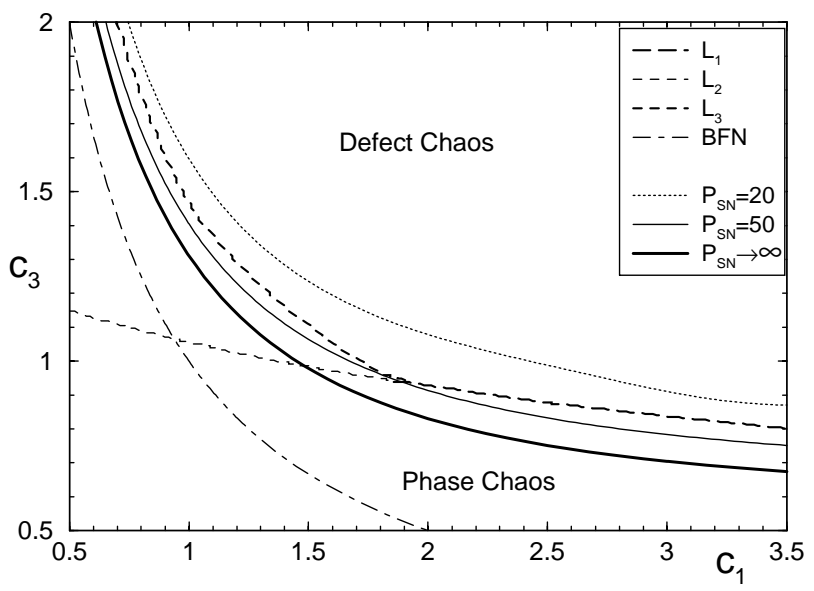

FIG. 1. Phase diagram of the CGLE showing the BFN, $L_{1}$, $L_{2}$, and $L_{3}$ transitions (after Ref. [7]). Between the $L_{2}$ and $L_{3}$ curves, there is the hysteretic regime where either phase or defect chaos can occur; in the latter case, defects persist up to the $L_{2}$ transition. Notice how the $L_{1}$ and $L_{3}$ transitions to defect chaos lie above our lower $(P \rightarrow \infty)$ bounds. Also shown are the SN locations for $P=20,50$. 
where $a$ and $\phi$ are real-valued functions of $z:=x-v t$. Such structures play an important role in various dynamical regimes of the CGLE [10-13]. The substitution of ansatz (2) into the CGLE leads to a set of three coupled ordinary differential equations (ODEs) for $a, b=d a / d z$, and $\psi=$ $d \phi / d z$ [14]. The MAWs correspond to limit cycles of these ODEs, or equivalently, spatially periodic solutions of the CGLE. The MAWs occur in a two parameter family which we choose to parametrize by their spatial period $P$ and their average phase gradient $\nu:=1 / P \int_{0}^{P} d z \psi$. Some examples of MAWs are shown in Figs. $2 b$ and 3. Only solutions for which $\nu=0$ are considered here; the reason for this will be discussed in (iii). To compute the MAWs and their bifurcations, we have used the software package AUTO94 [15] to solve the ODEs for fixed $P$ and $\nu$.

(ii) MAW range of existence.-MAWs with $\nu \neq 0$ bifurcate from unstable plane waves in the CGLE. We focus on the $\nu=0$ case, i.e., on the homogeneous oscillation $A(x, t)=e^{i c_{3} t}$. This solution becomes Benjamin-Feir (BF) unstable at $c_{1} c_{3}=1$, beyond which all plane waves are unstable [Benjamin-Feir-Newell (BFN) criterion] [1]. In the ODEs, the fixed point $(a, b, \psi)=(1,0,0)$ that corresponds to the homogeneous solution undergoes a Hopf bifurcation (HB) upon increasing $c_{1}$ and $c_{3}$. For infinite $P$ the Hopf bifurcation occurs for $c_{1} c_{3}=1$, while for smaller $P$ the Hopf bifurcation occurs for larger $c_{1}$ and $c_{3}$. The sequence of bifurcations for fixed $P=50$ is illustrated in Fig. 2a. The square symbol denotes the Hopf bifurcation, and the resulting solutions have drifting velocity $v=0$. Via a secondary drift pitchfork (DP) bifurcation [16] (diamond) the MAWs acquire $v \neq 0$. For the relevant parameters, i.e., sufficiently small $\nu$ and large $P$, both bifurcations are supercritical [2]; the amplitude modulations grow away from these bifurcations. The MAWs undergo a SN bifurcation (triangle) when $c_{1}$ or $c_{3}$ is sufficiently increased. The upper branch returns far back into the BF stable region of the CGLE; the recently discovered "homoclinic holes" [13] are MAWs of this upper branch in the limit $P \rightarrow \infty$. The spatial profiles of

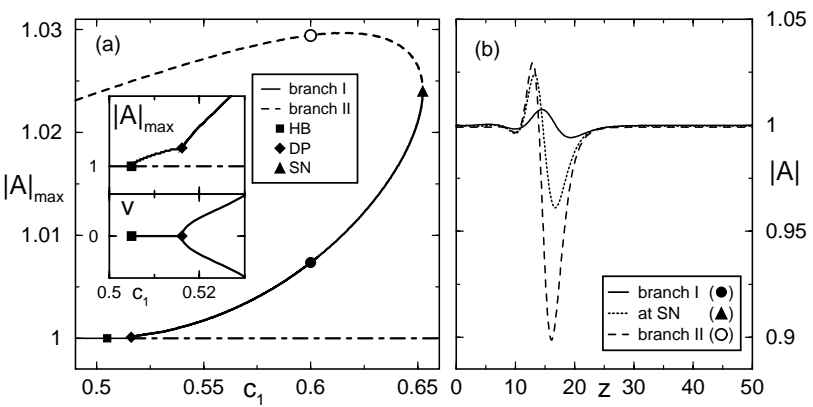

FIG. 2. (a) Example of the bifurcation diagram of MAWs for $c_{3}=2.0, P=50$ (see text). The inset illustrates the drift pitchfork bifurcation ( $v=0$ branch not shown beyond bifurcation). (b) MAW profiles at lower (full circle) and upper (open circle) branches, and at the SN (triangle).
MAWs on the upper (II) and lower (I) branches and SN are shown in Fig. 2b.

The SN curves in the $c_{1}-c_{3}$ parameter plane have been computed for various spatial periods $P$. For given parameters $c_{1}$ and $c_{3}$, we define $P_{\mathrm{SN}}$ as the period for which a SN bifurcation occurs. We find, roughly, that for larger $P$ this $\mathrm{SN}$ occurs for smaller values of $c_{1}, c_{3}$ (see Fig. 1).

To summarize: a family of coherent, periodic MAW solutions of the CGLE has been obtained. The range of existence of these solutions is limited by a SN bifurcation for large $c_{1}, c_{3}$.

(iii) Beyond the saddle node. - In Fig. 3 the relevance of the SN for defect generation is illustrated. In Fig. 3a we show the time evolution of a MAW-like initial condition in a periodic system of size $L>P_{\mathrm{SN}}$. While for $L<P_{\mathrm{SN}}$ we obtain coherent MAWs, for $L>P_{\mathrm{SN}}$ incoherent dynamics occurs: the amplitude modulation and drifting velocity grow until defects are formed. Extensive tests show that defects are always generated for MAW-like initial conditions when $L>P_{\mathrm{SN}}$. In Figs. $3 \mathrm{~b}$ and $3 \mathrm{c}$ the relevance of this defect generating mechanism for chaotic states is illustrated in a large system of size $L=512$ with coefficients close to the $L_{3}$ transition. The transient phase chaotic state (Fig. 3b) contains local structures which can come arbitrarily close to one-period MAWs. Figure $3 \mathrm{c}$ shows a snapshot of a spatial profile of $|A|$ in a phase chaotic state; parts of this profile can be approximated by a MAW with appropriate $P$. The phase gradient $\nu$ averaged between peaks of the amplitude is always close to zero; this is the reason why we focused on $\nu=0$ MAWs. Defects appear when one of these MAWs acquires a period larger than $P_{\mathrm{SN}}$ (Fig. 3b). This illustrates the main result: the transition to defect chaos occurs when a phase chaotic
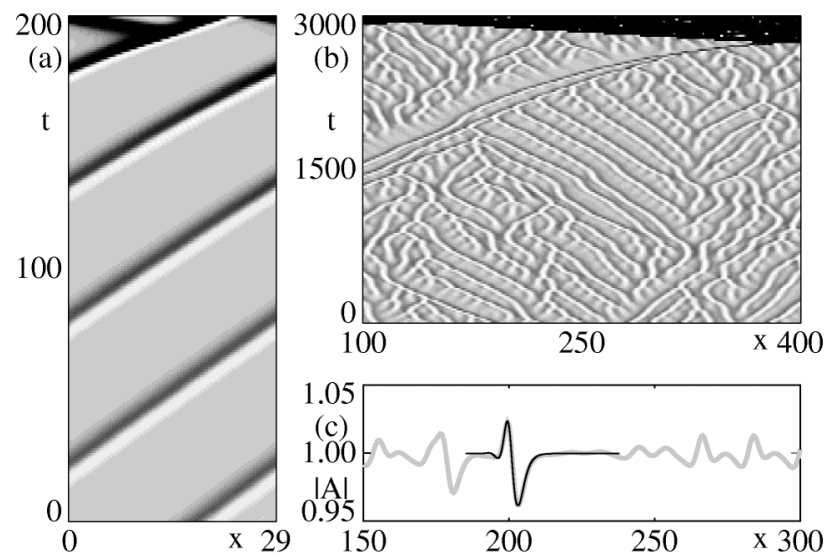

FIG. 3. (a) Grey-scale plot of $|A|$ (black: $|A| \rightarrow 0$ ) showing the nonlinear evolution of a near-MAW to defects when $L=$ $29>P_{\mathrm{SN}}=26.8\left(c_{1}=0.7, c_{3}=2\right)$. (b) MAWs and defect generation in a transient phase chaotic state $\left(c_{1}=0.66, c_{3}=\right.$ 2.0); a transient of $t \approx 10^{4}$ is not shown. (c) Comparison of a MAW (black line) with a snapshot from a phase chaotic state (grey line) $\left(c_{1}=0.65, c_{3}=2.0\right)$. 
state contains pulses with peak to peak distances larger than $P_{\mathrm{SN}}$.

To test the generality of this picture, we have carried out extensive numerical simulations of Eq. (1) near the transition lines $L_{1}$, respectively, $L_{3}$, adopting an integration algorithm developed in [11], in systems with sizes ranging from $L=100$ to $L=5000$ and integration times up to $5 \times 10^{6}$. The distribution of peak to peak distances $p$ of the phase gradients has been determined. Even though the phase chaotic state is not everywhere MAW like, we found that occurrences of large values of this "local" $p$ were approximated well by MAW profiles. Defects occurred in systems with $L \geq 512$ if and only if $p>P_{\mathrm{SN}}$. Since large $p$ 's are most "dangerous," the maximum value of $p, p_{\max }$, is the relevant quantity here. An example of $p_{\max }$ as a function of $c_{1}$ near $L_{3}$ is shown in Fig. 4 (squares); as soon as $p_{\max }$ crosses the $\mathrm{SN}$ curve, defects occur.

One may worry whether $p_{\max }$ is a well-defined quantity, especially in the thermodynamic limit. For larger system sizes and integration times $p_{\max }$ increases, however the apparent transition where defects occur shifts accordingly. For example, we found in our simulations that for $c_{3}=2.0$, the critical value of $c_{1}$ approximates 0.65 , while Ref. [7] finds, for shorter integration times, a critical value $\approx 0.68$. The fact that $p_{\max }$ (slowly) increases for larger systems/longer times is in agreement with earlier assertions that there is no sharp transition to defect chaos [9]. We have not been able to establish an upper bound for the $p$ 's occurring in phase chaos; therefore we conjecture that the SN line for $P \rightarrow \infty$ provides a lower boundary for the transition from phase to defect chaos.

(iv) MAW stability. - Of course, the laminar patches that occur in MAWs of large period are linearly unstable, and

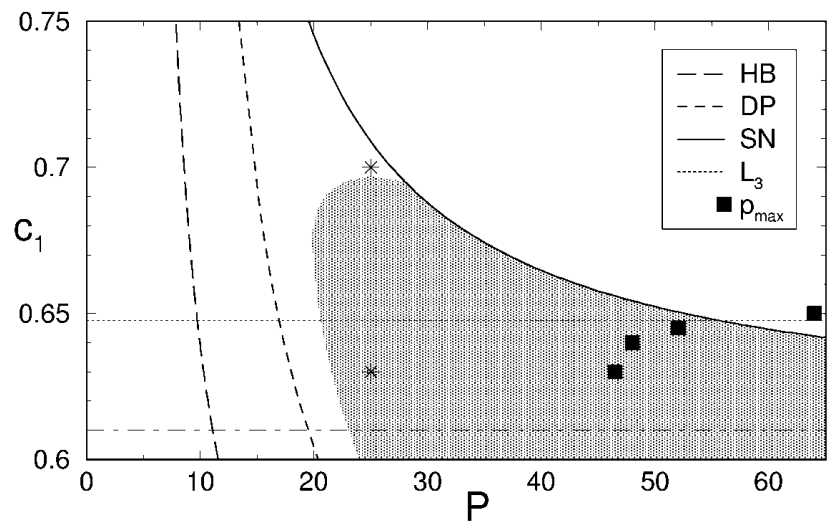

FIG. 4. Location of bifurcations and instabilities of MAWs as functions of $c_{1}$ and $P$ for $c_{3}=2.0$. Regions unstable to splitting modes are shaded. For large $P$, Hopf and drift pitchfork bifurcations approach the BFN line and the SN curve approaches $c_{1} \approx 0.61$ (dot-dashed line). The two stars mark parameters corresponding to stability spectra shown in Fig. 5. Black squares show the numerically measured maximum peak to peak distance $p_{\text {max }}$; once these squares cross the $\mathrm{SN}$ curve, defects occur. This is consistent with the numerically found location of $L_{3}$ (dotted line). large $P$-MAWs have only a small probability to occur. To get some further insight in the behavior of MAWs, we have calculated the linear stability properties of the MAWs. We start with a system of size $L=P$ and periodic boundary conditions. Both MAW branches have neutral modes corresponding to translational and phase symmetries. The eigenvalue associated with the $\mathrm{SN}$ is positive for solutions on branch II and negative for MAWs on branch I. Apart from these three purely real eigenvalues, the stability spectrum consists of pairs of complex conjugate eigenvalues.

In what follows the lower branch I is considered exclusively. For small enough $P$, all eigenvalues $\lambda$ have negative real parts, but when we increase $P$, MAWs become unstable to finite wave number perturbations. By using a Bloch ansatz, we extended the stability analysis to systems with $n$ identical pulses $(L=n P)$. For $n>1$, new instabilities may appear. The shape of these eigenmodes suggests that the instabilities lead to splitting of, respectively, interaction between adjacent MAWs; a nonlinear analysis confirms this. These instabilities are the relevant processes which locally decrease, respectively, increase $p$, thus inhibiting or enhancing the generation of defects. The splitting and interaction mechanisms are very similar to the cell splitting and instabilities one encounters in the Kuramoto-Sivashinsky equation [4].

The results of the stability analysis are summarized in Figs. 4 and 5. It is important to stress here that there is no qualitative difference between the behavior of MAWs near the $L_{3}$ and the $L_{1}$ transitions.

The eigenvalues with the largest real part on the connected curve in Figs. 5a and 5b correspond to "splitting" modes; Figs. $5 \mathrm{c}$ and $5 \mathrm{~d}$ display the nonlinear evolution that occurs when this mode is unstable. Clearly, this instability tends to reduce the spatial periods $p$ and prevents MAWs from crossing the SN boundary. Above a critical value for $c_{1}\left(c_{3}\right)$ the splitting modes are stable for all $P$ (Fig. 4). In this case the period of the MAWs can grow until $P>P_{\mathrm{SN}}$ is reached and defects are created.

The eigenvalues labeled by open squares in Figs. 5a and $5 \mathrm{~b}$ describe interaction between subsequent peaks that occurs for $n>1$ [17]. These interaction modes are mainly active for small $P$ (typically $P<20$ ). They cause instability of periodic MAWs and lead to local increase of the peak to peak distance $p$; Fig. 5e shows the nonlinear evolution in such a case.

Conclusion. - We have presented a systematic study of MAWs in the CGLE. These periodic coherent structures originate in supercritical bifurcations due to the $\mathrm{BF}$ instability of the CGLE. MAW existence is bounded by saddle-node bifurcations towards large $c_{1}, c_{3}$. Approaching the transition from phase to defect chaos, nearMAWs with large $P$ occur in phase chaos. Defects are generated if the period of these MAWs becomes larger than $P_{\mathrm{SN}}$. This scenario is valid for both the $L_{1}$ and $L_{3}$ transitions. Indications have been given in favor of the existence of the phase turbulent regime even in the 

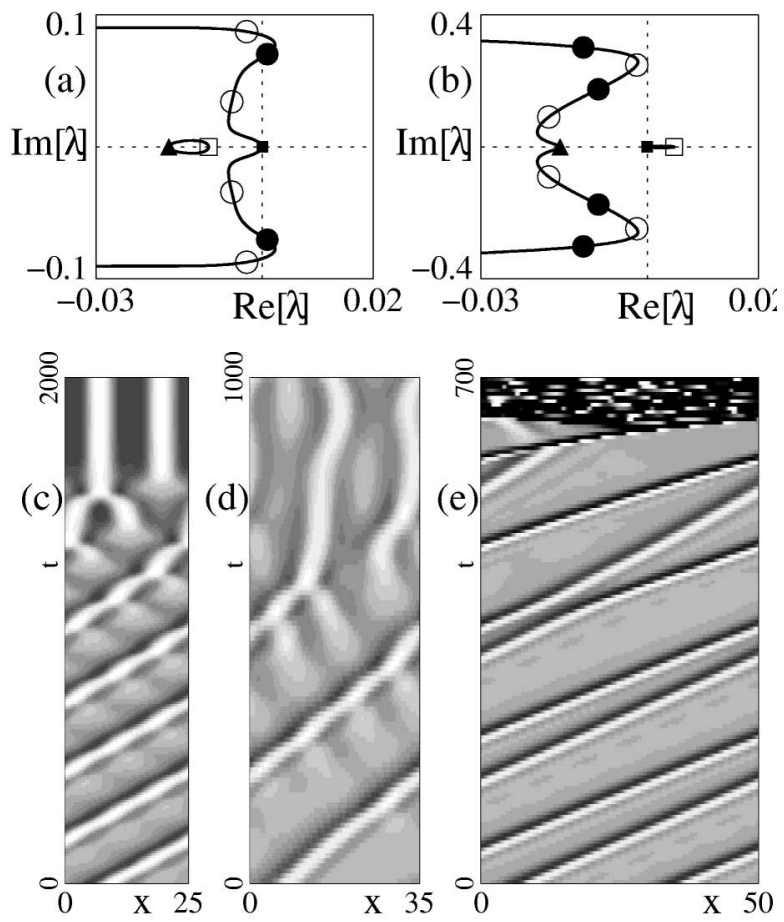

FIG. 5. (a),(b) Two typical stability spectra for $c_{3}=2.0$, $\nu=0, P=25$ and (a) $c_{1}=0.63$, respectively, (b) $c_{1}=$ 0.70. Filled symbols correspond to eigenvalues obtained for $L=P$, while open symbols denote additional eigenvalues for $L=2 P$ (symmetry modes: full square, splitting: circles, $\mathrm{SN}$ : triangle, interaction: open square); the curves show the spectrum for $L \rightarrow \infty$. (c),(d) Illustration of the splitting instability that decreases $p$ and prevents defects from occurring $\left(c_{1}=0.63\right)$. For small $L$ (c) the splitting leads to a stationary predrift pitchfork MAW, but for larger $L$ (d) disordered dynamics sets in. (e) Pulse interaction increases $p$ beyond $P_{\mathrm{SN}}$ and leads to defects $\left(c_{1}=0.7\right)$.

thermodynamic limit. Altogether, our study leaves little space for doubt that the transition from phase chaos to defect chaos in the CGLE is governed by coherent structures and their bifurcations.

It is a pleasure to acknowledge discussions with H. Chaté and L. Kramer. A.T. and M. B. are grateful to ISI Torino for providing a pleasant working environment during the Workshop on "Complexity and Chaos" in October 1999. M.G.Z. is supported by the MEC (Spain). M.v.H. acknowledges financial sup- port from the EU under Contract No. ERBFMBICT 972554.

*Present address: Physics Department, FCEN-University of Buenos Aires, Pab. I Ciudad Universitaria, (1428) Buenos Aires, Argentina.

[1] M. C. Cross and P. C. Hohenberg, Rev. Mod. Phys. 65, 851 (1993).

[2] B. Janiaud, A. Pumir, D. Bensimon, V. Croquette, H. Richter, and L. Kramer, Physica (Amsterdam) 55D, 269 (1992).

[3] N. Mukolobwiez, A. Chiffaudel, and F. Daviaud, Phys. Rev. Lett. 80, 4661 (1998).

[4] T. Bohr, M.H. Jensen, G. Paladin, and A. Vulpiani, Dynamical Systems Approach to Turbulence (Cambridge University Press, Cambridge, England, 1998).

[5] B. I. Shraiman, A. Pumir, W. van Saarloos, P.C. Hohenberg, H. Chaté, and M. Holen, Physica (Amsterdam) 57D, 241 (1992).

[6] M. Bazhenov, M. I. Rabinovich, and A. L. Fabrikant, Phys. Lett. A 163, 87 (1994).

[7] H. Chaté, Nonlinearity 7, 185 (1994); Spatio-Temporal Pattern Formation in Nonequilibrium Complex Systems, edited by P.E. Cladis and P. Palffy-Muhoray (Addison Wesley, Reading, 1995), p. 33.

[8] H. Sakaguchi, Prog. Theor. Phys. 84, 792 (1990).

[9] D. A. Egolf and H. S. Greenside, Phys. Rev. Lett. 74, 1751 (1995).

[10] R. Montagne, E. Hernández-García, and M. San Miguel, Phys. Rev. Lett. 77, 267 (1996); R. Montagne, E. Hernández-García, A. Amengual, and M. San Miguel, Phys. Rev. E 56, 151 (1997).

[11] A. Torcini, Phys. Rev. Lett. 77, 1047 (1996); A. Torcini, H. Frauenkron, and P. Grassberger, Phys. Rev E 55, 5073 (1997).

[12] W. van Saarloos and P.C. Hohenberg, Physica (Amsterdam) 56D, 303 (1992); 69D, 209 (1993) (Errata).

[13] M. van Hecke, Phys. Rev. Lett. 80, 1896 (1998).

[14] The three ODE system reads $a_{z}=b ; b_{z}=\psi^{2} a-$ $\gamma^{-1}\left[\left(1+c_{1} \omega\right) a+v\left(b+c_{1} \psi a\right)+\left(c_{1} c_{3}-1\right) a^{3}\right] ; \psi_{z}=$ $-2 b \psi / a+\gamma^{-1}\left[c_{1}-\omega+v\left(c_{1} b / a-\psi\right)-\left(c_{1}+\right.\right.$ $\left.\left.c_{3}\right) a^{2}\right]$. Abbreviations used are $\gamma=1+c_{1}^{2}, a_{z}=d a /$ $d z, b_{z}=d b / d z$, and $\psi_{z}=d \psi / d z$.

[15] E. J. Doedel, X. J. Wang, and T.F. Fairgrieve, AUTO 94, California Institute of Technology, 1994.

[16] M. Kness, L. S. Tuckerman, and D. Barkley, Phys. Rev. A 46, 5054 (1992).

[17] M. Or-Guil, I. G. Kevrekidis, and M. Bär, Physica (Amsterdam) 135D, 154 (2000). 